\title{
The efficacy of epiduroscopic adhesiolysis in patients with chronic back pain after surgery
}

\author{
Hakki OLCULU ${ }^{1}$ D, Kursat GUL ${ }^{1}$ D, Seniyye Ulgen ZENGIN² \\ ${ }^{1}$ Department of Anesthesia and Intensive Care, School of Medicine, Bezmialem Foundation University, Istanbul, Turkey. \\ ${ }^{2}$ Department of Anesthesia and Intensive Care, School of Medicine, Marmara University Teaching and Research Hospital, Istanbul, Turkey.
}

Corresponding Author: Seniyye Ulgen ZENGIN

E-mail: ulgen_t@yahoo.com

Submitted: 03.01.2021 Accepted: 27.04.2021

\begin{abstract}
Objective:Chronic back pain is a common problem with unwanted effects on the quality of life. The choice of treatment is usually patient-specific, but the use of epiduroscopic minimal invasive procedure is increasing. Epiduroscopy avoids surgical complications and improves patient comfort. This study was conducted to compare the efficacy of epiduroscopy in patients with failed back surgery syndrome (FBSS) and in patients without previous back surgery.

Patients and Methods: Forty-one ASA I-II-III patients aged 18-80 years old, with chronic back pain, radiologically and clinically diagnosed with lumbar spinal stenosis (LSS), and FBSS after laminectomy, hemilaminectomy, lumbar discectomy or lumbar spinal stabilization were included retrospectively. Patients were stratified as Group (O) with FBSS and Group (N) without previous back surgery. Baseline visual analogue scale (VAS) scores were obtained before treatment. During the 1st, 2nd and 3rd follow-ups, VAS scores of patients were measured.

Results: Visual analogue scale scores decreased significantly at 1,2, and 3 months after epiduroscopic adhesiolysis in both groups. The differences in VAS scores of patients with and without previous back surgery were not statistically significant.

Conclusion: Epiduroscopic adhesiolysis neuroplasty was followed by a significant decrease in chronic back pain in LSS and FBSS patients .

Keywords: Epiduroscopic adhesiololysis, Failed back surgery syndrome, Back pain
\end{abstract}

\section{INTRODUCTION}

Back pain is described as pain or muscle strain between the twelfth costal margin and the lower gluteal curve and can occur with or without leg pain. In $90 \%$ of patients, it resolves without treatment yet $10 \%$ have a chronic course of more than 12 weeks duration [1].The estimated prevalence of lifelong back pain is $60 \%-85 \%$, and it is the most common functional constraint and cause of lost work time among people younger than 45 years of age. The most important cause of back pain is lumbar spinal stenosis (LSS) resulting from lumbar spondylosis, ligamentum flavum hypertrophy, or spondylolisthesis. Many patients benefit from conservative treatment by epidural, transforaminal, or facet joint injection of drugs, but those are most often temporary solutions. Surgery may be indicated for non-responsive patients, but most are not candidates because of age or other factors. Epidural fibrosis, which may occur 6-12 weeks after back surgery, is another cause of back or leg pain $[2,3]$.Rapid pain relief often occurs after surgical procedures but can relapse because of fibrous adhesions [4]. Epiduroscopy is a novel, minimally invasive treatment of spinal pain. Its advantages include: 1) mechanical adhesiolysis with direct observation and laceration of adhesions and scar tissue; 2) saline infusion to dilute inflammatory mediators such as phospholipase-A2 and tumor necrosis factor; and 3) chemical adhesiolysis by injection 
of target-specific drugs. Epiduroscopy has been shown to reduce back pain and increase functional status [5,6-11].

Epiduroscopy was first used in the 1931s to demonstrate vertebral anatomy in cadavers. Clinical applications were recognized after Shimoji et al., described the use of conscious sedation and Saberski and Kitahata developed a caudal technique. Because patients under conscious sedation feel pain when their affected nerve roots are touched assists in identifying the area in which pain originates. The caudal technique minimizes complications such as dural perforation and headaches [12-14].

This study evaluated epiduroscopic adhesiolysis-neuroplasty in patients who visited an algology polyclinic complaining of back pain and were diagnosed with LSS with or without a history of back surgery.

\section{PATIENTS and METHODS}

Research Ethics Committee of Bezmialem Foundation University (no. 71306642-050.01.04) approved the study prior to initiation. American Society of Anesthesiologists (ASA) I-II-III patients between 18 and 80 years of age who visited the pain clinic for chronic back pain and consented to be treated by epiduroscopic adhesiolysis-neuroplasty. Written informed consent was obtained from all patients.

Patients with imaging evidence of pathologies that could cause LSS such as degenerative lumbar spondylosis, ligamentum flavum hypertrophy, or spondylolisthesis, or with back pain subsequent to back surgery performed at least 1 year previously and were not responsive to conservative medical therapy, physiotherapy, or 3 months of interventional injection treatment were included. Excluson criteria were, patients with systemic or local infections at the surgical location, coagulopathy, serious pulmonary disease, renal or liver failure, history of cerebrovascular events, intracranial masses that could increase the intracranial pressure, cauda equina syndrome, failed caudal interventions, experience respiratory or hemodynamic disturbance during the procedure. None of the subjects from both groups experinced any failed intervention or hemodynamic and respiratory problems. Study participants were stratified by their history to groups without (Group N), or with (Group O), previous back surgery. Participants were asked to indicate their pain on a vertical, numbered visual analogue scale (VAS) ruler $(0=$ no pain and 10 = worts pain ever had). Initial pain scores were determined with the VAS scale and recorded.

Patients fasted for at least 8 hours before starting the intervention. Venous access was established via the antecubital fossa with a $22 \mathrm{G}$ angiocath for premedication with $0.03 \mathrm{mg} / \mathrm{kg}$ midazolam. Patients were placed on the operating table in the prone position and monitored by electrocardiography, non-invasive blood pressure, and peripheral oxygen saturation. Fentanyl $1-2 \mathrm{mcg} / \mathrm{kg}$ and propofol $1-3 \mathrm{mg} / \mathrm{kg}$ conscious sedation was administered, and the sterile field was prepared. Local lidocaine anesthesia was applied to the insertion site and an 18 G Tuohy needle was inserted into the epidural space through the sacral hiatus. A floppy-tip guidewire (Tria V Guide, Tria Spine Med., Turkey) was passed into the epidural space and its location was confirmed by X-radiography. The site of guidewire insertion into the epidural space was incised and a $10 \mathrm{~F}$ dilatator was inserted by pushing it forward over the guidewire. After passing a $12 \mathrm{~F}$ valved introducer into the epidural space over the guidewire, the guidewire was removed and the epiduroscopy connections were made. To obtain a clear camera view and dilute inflammatory mediators, an isotonic $\mathrm{NaCl}$ solution was infused at $20-60 \mathrm{~mL} / \mathrm{min}$ depending on patient tolerability. After visual identification of the nerve roots affected by stenosis and the epidural adhesions, a mixture of $80 \mathrm{mg}$ methylprednisolone plus $40 \mathrm{mg}$ lidocaine was injected to the affected area. At the same time, mechanical adhesiolysis of the epidural adhesions was performed by gentle, controlled pressure using an epidural catheter. After completing these procedures, the operation was terminated by catheter removal and dressing and taping the insertion sites. The patients were routinely monitored for at least 1 hour in the recovery room before transfer to the service room. After discharge, patients were evaluated monthly for the next 3 months, including assessment of their VAS.

\section{Statistical Analysis}

Statistical analysis was performed with the Statistical Package for Social Sciences (SPSS). Data was tested for normal distribution by the Shapiro-Wilk test. Descriptive statistics were reported as averages \pm standard deviation for normally distributed continuous variables and as medians (minimummaximum) for the variables that were not normally distributed. Values of categorical variables were reported as frequency (n), and percentage (\%). The independent-sample $t$-test was used to determine the significance of differences in normally distributed continuous variables. The Mann-Whitney $U$ test was used to compare differences in independent variables that did not have a normal distribution; the Wilcoxon Signed Rank test was used with dependent variables. Pearson's chi-squared test was used to compare differences in the values of categorical variables. The significance level was designated as $\alpha=0.05$. $p$-values $<0.05$ were considered significant. Significant differences are indicated in bold text.

\section{RESULTS}

Forty-one patients with LSS and epiduroscopic adhesiolysisneuroplasty were enrolled between June 1 and August 2, 2016. Twenty-one patients were included in Group $\mathrm{N}$ (without back surgery) and 20 in Group $\mathrm{O}$ (with back surgery). The patient characteristics are shown in Table I. There were no significant differences in the age and sex ratio of the two groups, which were considered as a homogeneous population.

The initial VAS scores and the scores obtained during follow-ups after the epiduroscopic procedure are shown in Table II. After epiduroscopic adhesiolysis-neuroplasty, there was a significant decrease in the back pain scores reported by the patients in both study groups throughout the follow-up period.

The initial and follow-up VAS scores of both groups are shown and compared in Table II 
As noted above, the initial scores in patients with a history of back surgery and in those without previous back surgery were not significantly different. Within each group, there was a significant decline in VAS score following epiduroscopy. However, there were no significant differences between two groups ( $p>0.05$ ), indicating that the effectiveness of the procedure in each group was similar.

Table I. Patients Baseline Characteristics

\begin{tabular}{|l|l|l|}
\hline Demographic Data & $\begin{array}{l}\text { Group N } \\
(\mathbf{n = 2 1 )}\end{array}$ & $\begin{array}{l}\text { Group O } \\
(\mathbf{n = 2 0 )}\end{array}$ \\
\hline Age (average \pm SD) & $66.05 \pm 8.880$ & $61.75 \pm 10.622$ \\
\hline Female Gender n (\%) & $12(57.1 \%)$ & $8(40 \%)$ \\
\hline Male Gender $\mathrm{n}(\%)$ & $9(42.9 \%)$ & $12(60 \%)$ \\
\hline
\end{tabular}

Table II. VAS scores and between-group differences in patients with (Group O) and without (Group N) previous back surgery

\begin{tabular}{|c|c|c|c|c|c|c|c|c|}
\hline Groups & & Initial VAS & 1MonthVAS & 2MonthVAS & 3MonthVAS & $\begin{array}{l}\text { Differ. } \\
1\end{array}$ & $\begin{array}{l}\text { Differ. } \\
2 \\
\end{array}$ & $\begin{array}{l}\text { Differ. } \\
3 \\
\end{array}$ \\
\hline \multirow[t]{6}{*}{1 Non - Operated } & $\mathrm{n}(21)$ & & & & & & & \\
\hline & Median & 8.00 & 4.00 & 3.00 & 2.00 & -3.00 & -4.00 & -4.00 \\
\hline & Minimum & 5 & 0 & 0 & 0 & -8 & -9 & -9 \\
\hline & Maximum & 10 & 8 & 8 & 8 & 0 & 0 & 0 \\
\hline & Mean & 7.81 & 4.52 & 3.48 & 3.24 & -3.29 & -4.33 & -4.57 \\
\hline & Std. Deviation & 1.209 & 2.228 & 2.379 & 2.406 & 2.348 & 2.497 & 2.561 \\
\hline \multirow[t]{6}{*}{2 Operated } & $\mathrm{n}(20)$ & & & & & & & \\
\hline & Median & 8.00 & 4.00 & 2.50 & 2.50 & -4.00 & -4.50 & -4.50 \\
\hline & Minimum & 5 & 2 & 0 & 0 & -7 & -8 & -9 \\
\hline & Maximum & 10 & 8 & 8 & 8 & 0 & 0 & 0 \\
\hline & Mean & 7.70 & 4.20 & 3.45 & 3.10 & -3.50 & -4.25 & -4.60 \\
\hline & Std. Deviation & 1.302 & 1.989 & 2.544 & 2.654 & 2.188 & 2.673 & 2.854 \\
\hline $\mathrm{p}$ & & 0.751 & & & & 0.634 & 0.948 & 0.937 \\
\hline
\end{tabular}

\section{DISCUSSION}

Chronicback pain is a significant psychosocial problem, especially in the elderly population. It is also a major socioeconomic problem limiting physical activities of people under 45 years of age. Back surgery may result in the development of severe scarring of dermal, subcutaneous, and connective tissues. The scar tissue can restrict and inhibit movements of the affected region producing generalized pain or fibromyalgia. Fibrous epidural scar tissue present in FBSS patients can cause nerve entrapment, stenosis, and functional movement limitations that cannot be detected by magnetic resonance imaging (MRI) [8]. Many patients who suffer from chronic back pain are elderly and relatively less tolerant of general anesthesia and major surgical interventions. However, surgery is indicated in some conditions such as cauda equina syndrome or sequestered disks [4].

Epiduroscopy is an endoscopic procedure for patients with back pain unresponsive to physical therapy, drugs, or transforaminal injections. It has important advantages in determining the cause of pain, mechanically separating adhesions, and injecting medications to the target regions [14-18]. Bosscher and Heavner confirmed the high sensitivity of epiduroscopy by demonstrating its efficacy in diagnosing epidural fibrosis in a series of FBSS patients, compared to MRI (91\% versus $16.1 \%$, respectively) [11]. Similarly, in a prospective study concerning target-specific adhesiolysis and epidural injections for relieving radicular pain, this procedure identified epidural adhesions in 19 of 20 patients [7]. Nevertheless, the adhesions could not be detected by MRI, in 8 of the 19 patients [7]. Manchikanti et al., compared the effectiveness of conventional chemical adhesiolysis using steroids and local anesthetics to detect lesions, with or without epiduroscopy [12]. Without the aid of epiduroscopy, adhesiolysis was repeated six times in one, five times in three, and four times in twelve patients. With epiduroscopy, adhesiolysis was performed three times in only one patient during 12 months of follow-up. Effectiveness was defined as more than $50 \%$ decrease in pain, and after the first epiduroscopy procedure, a significant decrease in pain was observed in all patients. The comparison of non epiduroscopic adhesiolysis with the epiduroscopic one, revealed that the rate of decrease in pain was $72 \%$ versus $97 \%$ in the first month, $25 \%$ versus $80 \%$ in the third month, $10 \%$ versus 
$52 \%$ in the sixth month, and $7 \%$ versus $22 \%$ in the twelfth month of the procedure, respectively. The results were also comparable after the second procedure. It was concluded that epiduroscopic adhesiolysis had largely positive effects. [12]. In our study we found positive effects on VAS values at each of the 3 months of patient follow up in both operated and non operated groups.

Pereria et al., evaluated the effects of adhesiolysis under epiduroscopy in patients with back or leg pain, unresponsive to the conservative therapy for six months, after lumbar discectomy [14]. Saline infusion was used for soft adhesions and mechanical pressure was applied to hard fibrous tissues using a catheter. Radiofrequency ablation was applied to septas that remained despite those procedures. After adhesiolysis, betamethazone and bupivacaine were injected. to each patient. Effectiveness was assessed by VAS and Oswestry Disability Index 2.0 scores. Significant decreases in VAS scores were seen in $71 \%$ of the patients in the first month, in $63 \%$ between 3 and 6 months, and in $38 \%$ at 12 months after treatment. The decreases in back and/or leg pain were statistically significant [14]. Rafaelli and Righetti have reported successful lysis of adhesions in 14 patients with a $4 \mathrm{Mhz}$ Res-Ablator and over $90 \%$ recovery in eight of the patients (57\%) [15].

In FBSS patients, epiduroscopy can be used in the lysis of adhesions caused by fibrous scar tissues (adhesiolysis). In LSS patients it can improve the physiological activity of nerve roots and the spinal cord (neuroplasty) by reducing the pressure on the medulla spinalis and nerve roots that results from stenosis. Jo et al., retrospectively evaluated epiduroscopy and ELND (Epiduroscopic laser neural decompression) in 39 patients with characteristics similar to the those enrolled in this study. Laser ablation was applied to pain-causing lesions visualized by epiduroscopy. The patients had not responded to previous medical treatment, epidural steroid injection, or surgery, or were patients with chronic back pain who relapsed 1 week after previous treatment. Some patients had requested ELND as initial treatment. Patient satisfaction was scored as "good," "acceptable," or "bad." Seventeen patients had previous lumbar surgery and 22 did not. Sixteen (94.1\%) surgical patients and 19 nonsurgical patients $(86.3 \%)$ reported that the study treatment outcome was acceptable or good. The difference was not significant but the improvement of chronic back and leg pain was judged clinically significant in both groups. [16]. The results of this study are in line with those of Jo et al., in that epiduroscopic adhesiolysisneuroplasty was effective in LSS patients with and without FBSS [16].

Complications of epiduroscopic adhesiolysis include pain at the insertion site, dural-perforation headache, infection, cerebrospinal fluid pressure caused by bolus injection. neurologic sequelae caused by epidural hematoma, and steroid side effects $[6,7,20]$. Although, case studies have shown epiduroscopic adhesiolysis to be a medically safe procedure, some serious complications have been reported [22,23]. No complications occurred in our study. Even though the patient number is statistically adequate to them, the sample size and relatively short follow-up can be seen as limitations. Unfortunately, the patient portfolio in our country does not include very much data on complications compared with controls beyond that needed for a conclusion of adequate safety.

The study evaluated and compared the effectiveness of epiduroscopic adhesiolysis-neuroplasty in FBSS and LSS patients. FBSS is characterized by post-surgical pain caused by pressure on spinal nerves following development of fibrous epidural scar tissue 6-12 weeks after the procedure. The pressure disturbs the physiological activities of nerves. FBSS can occur even after technically and anatomically proper surgical procedures. Mechano-chemical laceration or disruption of epidural fibrosis reducing the pressure on nerves and thus relieves the back pain. Because of this, we expected this procedure to be more effective in FBSS than in LSS patients.

\section{Conclusion}

Epiduroscopic adhesiolysis-neuroplasty was effective in decreasing chronic back pain in both LSS patients and FBSS patients with previous back surgery including laminectomy, hemilaminectomy, lumbar discectomy, or posterior stabilization. Considering FBS, epiduroscopic adhesiolysis may be a good choice in appropriate cases with LSS since, it is an effective and a minimally invasive method.

\section{Compliance with Ethical Standards}

Ethical Approval: This study was approved by the Ethics Committee of Bezmialem Foundation University (no. 71306642050.01.04). Written informed consent was obtained from all patients before the procedure.

Funding: The authors have no relevant financial information to disclose.

Conflict of interest: The authors declare no conflict of interest.

Authors' Contrubition: I. H. O., K. G., S.U.Z Idea/ Concept. K.G.,S.U. Z: Design. K.G.,S.U. Z. Control/Supervision. I.H.O., K.G.,S.U. Z. Data Collection and processing. I.H.O., K.G,S.U. Z.Analysis. I.H.O., K.G.,S.U. Z. Literature review. S.U.Z/ K.G. Critical Review. All authors approved the final version of the article.

\section{REFERENCES}

[1] Henschke N, Maher CG, Refshauge KM, et al. Prevalence of and screening for serious spinal pathology in patients presenting to primary care settings with acute low back pain. Arthritis Rheum 2009; 60:3072-80. doi:10.1002/art.24853

[2] Blomberg RG, Olsson SS. The lumbar epidural space in patients examined with epiduroscopy. Anesth Analg 1989; 68:157-60. doi:10.1213/00000.539.198902000-00016

[3] Hussain A, Erdek M. Interventional pain management for failed back surgery syndrome. Pain Pract 2014; 14:64-78. doi:10.1111/papr.12035

[4] Weinstein JN, Tosteson TD, Lurie JD, et al. Surgical versus nonsurgical therapy for lumbar spinal stenosis. N Engl J Med 2008; 358:794-810. doi:10.1056/NEJMoa0707136 
[5] Manchikanti L, Boswell MV, Rivera JJ, et al. A randomized, controlled trial of spinal endoscopic adhesiolysis in chronic refractory low back and lower extremity pain. BMC Anesthesiol. 2005; 5:10. doi:10.1186/1471-2253-5-10

[6] Geurts JW, Kallewaard JW, Richardson J, Groen GJ. Targeted methylprednisolone acetate/hyaluronidase/clonidine injection after diagnostic epiduroscopy for chronic sciatica: a prospective, 1-year follow-up study. Reg Anesth Pain Med 2002; 27:343-52. doi:10.1053/rapm.2002.27175

[7] Sakai T, Aoki H, Hojo M, et al. Adhesiolysis and targeted steroid/local anesthetic injection during epiduroscopy alleviates pain and reduces sensory nerve dysfunction in patients with chronic sciatica. J Anesth. 2008;22(3):242-247. doi:10.1007/s00540.008.0616-4.

[8] Avellanal M, Diaz-Reganon G, Orts A, Gonzalez-Montero L, Riquelme I. Transforaminal epiduroscopy in patients with failed back surgery syndrome. Pain Physician2019; 22:89-95.

[9] Geudeke MW, Krediet AC, Bilecen S, Huygen FJPM, Rijsdijk M. Effectiveness of epiduroscopy for patients with failed back surgery syndrome: a systematic review and meta-analysis. Pain Pract 2021; 21:468-1. doi: 10.1111/papr.12974.

[10] Saberski LR, Kitahata LM. Direct visualization of the lumbosacral epidural space through the sacral hiatus. Anesth Analg 1995; 80:839-40. doi:10.1097/00000.539.19950400000035

[11] Bosscher HA, Heavner JE. Incidence and severity of epidural fibrosis after back surgery: an endoscopic study. Pain Pract 2010; 10:18-24. doi:10.1111/j.1533-2500.2009.00311.x

[12] Manchikanti L, Cash KA, McManus CD, Damron KS, Pampati V, Falco FJ. A randomized, double-blind controlled trial of lumbar interlaminar epidural injections in central spinal stenosis: 2-year follow-up. Pain Physician 2015; 18:79-92.

[13] Lodh M, Goswami B, Mahajan RD, Sen D, Jajodia N, Roy A. Assessment of Vitamin D status of chronic in patients low back pain of unknown etiology. Indian J Clin Biochem 2015; 30:174-9. doi:10.1007/s12291.014.0435-3
[14] Pereira P, Severo M, Monteiro P, et al. Results of lumbar endoscopic adhesiolysis using a radiofrequency catheter in patients with postoperative fibrosis and persistent or recurrent symptoms after discectomy. Pain Pract 2016; 16:6779. doi:10.1111/papr.12266

[15] Raffaeli W, Righetti D. Surgical radio-frequency epiduroscopy technique (R-ResAblator) and FBSS treatment: preliminary evaluations. Acta Neurochir Suppl 2005; 92:121-5. doi:10.1007/3-211-27458-8_26

[16] Jo DH, Kim ED, Oh HJ. The Comparison of the result of epiduroscopic laser neural decompression between FBSS or not. Korean J Pain 2014; 27:63-7. doi:10.3344/kjp.2014.27.1.63.

[17] Chopra P, Smith HS, Deer TR, Bowman RC. Role of adhesiolysis in the management of chronic spinal pain: a systematic review of effectiveness and complications. Pain Physician 2005; 8:87-100.

[18] Saberski LR. A retrospective analysis of spinal canal endoscopy and laminectomy outcomes data. Pain Physician 2000; 3:193-6.

[19] Manchikanti. L., Pakanati R.R., Pampati V, Fellows B. Review papers-the value and safety of epidural endoscopic adhesiolysis-epidural endoscopy has been considered an expensive conservative modality; this study evaluated its overall effectiveness. Am J Anesthesiol 2000; 27: 275-82.

[20] Gill JB, Heavner JE. Visual impairment following epidural fluid injections and epiduroscopy: a review. Pain Med 2005; 6:367-74. doi:10.1111/j.1526-4637.2005.00062.x

[21] Manchikanti L. Role of neuraxial steroids in interventional pain management. Pain Physician 2002; 5:182-99.

[22] Heavner J E, Wyatt DE, Bosscher HA. Lumbosacral epiduroscopy complicated by intravascular injection. The Journal of the American Society of Anesthesiologists KISA HALI'? 2007; 107:347-50.

[23] Marchesini M, Flaviano E, Bellini V, Baciarello M, Bignami EG. Complication of epiduroscopy: a brief review and case report. Korean J Pain 2018; 31:296-304. doi:10.3344/ kjp.2018.31.4.296 